\title{
Artificially generated cultural variation between two groups of captive monkeys, Colobus guereza kikuyuensis
}

\author{
Elizabeth Price \\ Christine A. Caldwell \\ School of Psychology, University of Exeter, UK.
}

\begin{abstract}
The majority of studies of social learning in primates have tested subjects in isolation and investigated the effects of learning over very short periods of time. We aimed to test for social learning in two social groups of colobus monkeys, Colobus guereza kikuyuensis. Subjects were shown video footage of familiar monkeys either pushing or pulling a plastic flap to obtain a food reward, while they were given simultaneous access to the same apparatus. Action frequencies showed a significant difference between the two groups, with the pull group performing a higher proportion of pulls to pushes, compared with the push group. Copying persisted even in later sessions during which the demonstration footage was not being shown. We conclude that we successfully generated two contrasting behavioural traditions in these groups of monkeys. We do not know how long this contrast in behaviour would have persisted had we been able to continue testing for an even longer period of time, but further studies using similar designs and even longer test periods would have the power to confirm whether stable behavioural variation can be sustained between groups of monkeys, supported by social transmission.
\end{abstract}

\section{Introduction}

There is now increasing evidence for cultural variation in primate behaviour, based on systematic surveys of different field sites. Researchers have shown that behaviours that are commonly observed at some field sites are absent at others, without any apparent ecological explanation (chimpanzees - Whiten et al, 1999; 2001; orangutans - van Schaik et al 2003; capuchin monkeys - Perry et al, 2003; Panger et al, 2002). Nonetheless, some researchers still express scepticism about whether such variation is genuinely cultural, in the sense of being supported by social transmission (Laland \& Hoppitt, 2003; Galef, 2003).

The experimental literature would suggest that primates are capable of fairly complex social learning. Studies using the "two-action" method (Dawson and Foss, 1965) have shown that subjects are influenced by the particular behaviours they have seen demonstrated (e.g. chimpanzees - Whiten et al, 1996; capuchins - Custance et al, 1999; marmosets - Voelkl \& Huber, 2000). Contrasting behaviours can therefore be induced in primates through observation of conspecifics.

However, these studies each tested individuals separated from their social group, and tested the effects of the demonstration on behaviour over only very short periods of time. Whiten et al. (1996), Custance et al. (1999), and Voelkl and Huber (2000) all describe clearly in their methods sections the delay between demonstration and trials, and in each case, subjects were tested for only a matter of minutes following the demonstrations. The typically short duration of such studies has meant that we currently know little about how long socially learned behaviours will remain in primates' repertoires, especially once subjects have been given an opportunity to interact with the relevant objects themselves. Such experience may allow individuals to discover alternative (and possibly more efficient) solutions through their own 
experiences, which may quickly obscure any initial differences in behaviour, causing two initially divergent groups to rapidly converge on the more efficient of the two solutions.

Using this kind of logic, Galef (1995) argued that those behaviours that are maintained in populations (such as those commonly labelled as culture in primates) must be locally adaptive, in the sense that they must result in greater reinforcement relative to alternatives. According to this argument, divergent patterns of behaviour observed at different field sites almost certainly have their origins in environmental shaping, rather than cultural variation. Heyes (1993) put forward a similar argument, citing Galef et al's (1986) data from a two-action study on budgerigars: subjects that observed conspecifics accessing hidden food using either their beak or their foot tended to match the demonstrated technique for only the first two trials postdemonstration. In later trials, the difference between the groups disappeared.

Recently, Whiten et al (2005) attempted to artificially induce cultural variation in two groups of chimpanzees. Two demonstrator chimpanzees, one from each of the social groups, were trained to perform a tool-use task, each using a different method from the other. When the two groups had the opportunity to observe their demonstrator operating the apparatus, there was a clear tendency for subjects to use the method that they had seen. Furthermore, the variation between the two groups was maintained over several days of testing, during which the apparatus was available to the chimpanzees for a total of 36 hours. Fourteen out of the 32 subjects in fact showed an increasing tendency to use the "group norm", despite having also used the alternative. Only two chimpanzees used the alternative method more than the demonstrated technique. This study effectively showed that social learning in primates does have the capacity to support natural behavioural variation, as the researchers showed that these alternatives were maintained over time within the two social groups.

The aim of the current study was to investigate social learning in a similar manner in a monkey species. We used a two-action task to test two captively housed groups of Abysinnian colobus monkeys (Colobus guereza kikuyuensis) at Paignton Zoo Environmental Park. Little is known about the cognitive abilities of Abyssinian colobus monkeys despite their increasing popularity in zoos. Colobus monkeys are primarily dependent on leaves, and it has been hypothesized that the relative abundance and wide-spread dispersal of their diet leads to little conflict within the species (Davies \& Oates, 1994; Walker \& Murray, 1975). Their relatively high levels of social tolerance might therefore be expected to make them good candidates for social learning (Coussi-Korbel and Fragaszy, 1995).

It was decided that a "virtual demonstrator" (Mottley \& Heyes, 2003) methodology would be a particularly useful for testing in the zoo environment. Mottley and Heyes (2003) used this method as a means of controlling imitation studies more effectively than is possible with live animal demonstrators. They devised a method by which observer budgerigars were shown video footage of conspecific demonstrators using their beaks to remove a stopper from a food box by either pulling or pushing the object. This design not only controls for the effects of discrepancies in demonstration techniques, but also opens a new realm of possibilities for social learning research, in terms of studying animals that cannot be tested under normal laboratory conditions. Video images have been used in place of live animal stimuli in a wide range of experiments, with a variety of different species, including primates (D’eath, 1998). Cook and Mineka (1989) showed that rhesus monkeys (Macaca mulatta) learn to react fearfully to snakes after watching video footage of another 
monkey reacting in the same manner. Some studies have found problems with the use of video images in behavioural experiments, but these have typically been attributable to problems of flicker associated with the use of standard cathode ray tube monitors, combined with a high critical flicker frequency in the species being tested. Liquid crystal displays, which produce no flicker, have however generally been found to be effective (e.g. see Ikebuchi \& Okanoya, 1999, for a direct comparison of the two methods).

The apparatus used in the current study was a basic push or pull two-action task, similar to that used by Bugnyar and Huber (1997). The method involved capturing footage of a group of king colobus monkeys (Colobus polykomos, also housed at Paignton Zoo) solving the task two different ways. Edited footage of the two alternative solutions could then be shown to different groups of Abyssinian colobus monkeys. The monkeys were to be tested on a total of ten sessions, each held on different days. It was predicted that there would be a significant difference between the groups in terms of the number of pushes to pulls that the subjects performed, with subjects tending to match the version that they had seen demonstrated.

\section{Methods}

\section{Subjects}

The subjects consisted of two groups of Abyssinian colobus monkeys (Colobus guereza kikuyuensis) housed at Paignton Zoo and Environmental Park. The first group was comprised of four females: two mothers aged 11 and 9 (Diamond and Beattie), and their respective offspring both 3.5 years old (Scragg and Lottie). The group was provided with indoor and outdoor access linked by a caged corridor. Both areas of the enclosure were visible to the public. The second group of monkeys was made up of six males and two females. Only three of these monkeys participated however: Kabul, Hope, and Fergus, all male juveniles of 2.5 years, 1.5 years, and 3.5 years old respectively, and all the offspring of one of the females in the same group. They were situated across a public viewing gallery from the other group and housed in a similar indoor/outdoor enclosure. The second group regularly participated in operant conditioning procedures to assist in veterinary care, and both groups were provided with weekly enrichment activities.

A group of king colobus monkeys (Colobus polykomos) also housed at Paignton Zoo were used to create the demonstration footage. The group consisted of two males and four females, housed adjacent to the small group of Abyssinian colobus monkeys. Of these, three monkeys participated, although footage of only one female was used in the demonstration clips. Although the demonstrator monkeys were of a different species, at the zoo they were in daily visual contact with the Abyssinian colobus monkeys that were used as subjects, and in the wild the two species are known to gather in interspecies groups, often feeding from the same sites (Davies and Oates, 1994).

All animals, both subjects and demonstrators, had been kept in captivity at Paignton Zoo for the duration of their lives. They were fed a small amount of food between 09:00h and 10:00h in the morning, and then fed their primary meal at between $15: 00 \mathrm{~h}$ and $15: 30 \mathrm{~h}$ in the afternoon. Their diet consisted largely of vegetables and small portions of wheat bread, as well as leafy branches. 


\section{Apparatus}

The apparatus was a square metal box, $16 \mathrm{~cm}$ long by $16 \mathrm{~cm}$ wide and $16 \mathrm{~cm}$ deep. A $10 \mathrm{~cm}$ by $16 \mathrm{~cm}$ hole in the front of the box was covered by a transparent, flexible plastic flap secured by two bolts. Two metal hooks were welded onto the front of the box, and two adjustable hooks attached to the sides to enable the apparatus to hang on the cage. A door in the rear of the apparatus could be opened by removing a single screw. Food was placed through a small $2.5 \mathrm{~cm}$ circular hole cut into the upper right hand of the rear of the box.

Holes measuring approximately $18 \mathrm{~cm}$ by $18 \mathrm{~cm}$ were cut into the wire mesh of each of the enclosures so the subjects would have direct access to the box from inside the enclosure. In between sessions, the apparatus was removed and a small patch was placed in front of the hole, secured at the top with metal clips and at the bottom with several large cable ties. At the start of a session, the bottom cable ties were removed and the patch was flipped upwards to allow for the attachment of the apparatus.

The plastic flap at the front of the box could be pushed in or pulled out to retrieve the food reward. Several different handles were tested on the king colobus monkeys in order to establish a relatively simple method of pulling. The handle had to be constructed both in consideration of the subjects' manipulative abilities, as well as the safety approval of the zoo. A bolt was secured at the bottom centre of the flap to give the monkeys something to pull up on, however this was rarely used in preference for pulling up on the edge and corner of the flap.

\section{Demonstration Videoclips}

Recording of demonstration videoclips took place opportunistically over the course of one week. The box was attached to the outside king colobus enclosure at a point not visible to either of the observation groups. Subjects were given free access to the box across seven 20-minute sessions as previously described. Every time a piece of food was successfully removed from the box, a new one was replaced. The subjects were not trained, and therefore the actions recorded were spontaneous, occurring through trial-and-error manipulations with the box. All interactions were recorded using a Panasonic digital video camera attached to a tripod and placed about half of a metre outside of the cage, approximately one metre from the apparatus. This allowed for a clear side view of the demonstrator monkey performing the task. Once clear examples were obtained of both push and pull solutions, the footage was reviewed and edited to create two demonstration clips, one illustrating the push solution and one the pull solution. In order to match the clips as closely as possible, examples from the same female demonstrator were chosen for both clips. Each clip consisted of two successive successful attempts to retrieve the food and lasted approximately 10 seconds. Stills taken from the pull and push demonstrations can be found in Figure 1. In the push solution clip the demonstrator pushed through the flap with her hand and removed the food with the same hand, and in the pull solution clip she lifted the flap up from the bottom corner with one hand, and reached in to retrieve the food with the other hand. 


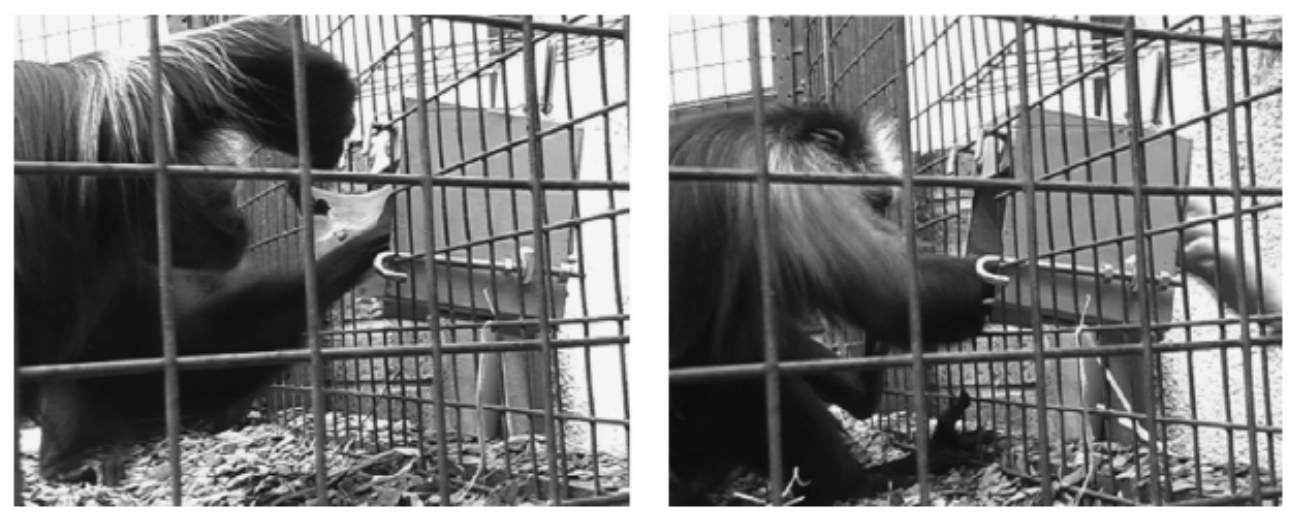

Fig. 1. Stills from the pull (left) and push (right) demonstrations.

\section{Procedure}

Demonstration type was assigned to the two observation groups at random. The demonstration footage was transferred onto a CD in the form of two digital QuickTime movies, and played on a battery powered Dell laptop with an LCD display. The laptop was placed half of a metre from the edge of the cage, in close proximity to where the apparatus would later be attached. The visibility of the demonstration footage was checked by the experimenter, who entered the two enclosures whilst the animals were separated off, and verified that the view from within the enclosure was adequate, particularly from the point of view of a subject who would be operating the apparatus.

Each group was exposed to a continuous loop of the appropriate demonstration footage without access to the apparatus for two initial twenty-minute sessions. This was both to reduce any possible inhibition effects caused by introduction of the video footage, as well as to ensure that each subject was exposed to the chosen demonstration before interacting with the apparatus. This occurred during the days immediately preceding the experimental sessions.

Ten experimental sessions took place across three weeks; eight sessions including simultaneous demonstration footage of the solution, and two follow-up sessions with no demonstration. Each session lasted 30 minutes and took place in the morning between 08:00h and 10:30h, during the monkeys' morning feed, before the public had access to the zoo. Due to the requirements of the zookeepers, and to ensure that the two groups did not have visual access to each other performing the task, it was necessary to install the apparatus in the outdoor section of the "pull" group, and in the indoor section of the "push" group enclosure. For the pull group, the apparatus was placed at the rear of the outdoor enclosure, away from the public viewing area, approximately $30 \mathrm{~cm}$ above the ground. The laptop was placed adjacent to the apparatus so that the subjects could interact with the box, while simultaneously viewing the demonstration footage. The same Panasonic video recorder was used to document all interactions, set up on a tripod approximately one metre from the apparatus, giving a side view of the monkeys as they interacted with the boxes. For the push group, the apparatus was attached in the indoor enclosure, again at a point to which the public had no access, approximately $60 \mathrm{~cm}$ above the ground. The laptop was placed adjacent to and level with the apparatus, as with the other group. All 
interactions were filmed through the public viewing glass, allowing for a direct view of the monkeys interacting with the apparatus.

All subjects had simultaneous access to the apparatus, and the demonstration footage ran for the duration of the sessions. Demonstration footage was not shown during the two follow-up sessions. The ninth session occurred on the day directly after the eighth session, the tenth session occurred five days after the ninth.

\section{Coding and Reliability}

All four monkeys in the pull group participated (two juvenile females and two adult females), whereas only three of the seven monkeys from the push group participated (all three were juvenile males). Individuals were considered to have participated if they made any attempt at a push or pull. Those individuals who did not participate in the large group did not show any interest, with the exception of Erroll, an adult male, who occasionally watched the others interacting with the apparatus. Frequencies of all successful and unsuccessful pushes and pulls were tallied for both groups using slow-motion playback. A successful pull was classified as a subject pulling the flap outward by whatever method (lifting at the corner or edge of the flap, or biting or pulling up on the bolt) and successfully retrieving a piece of bread. Similarly, a successful push was recorded when the flap was pushed inward and a piece of food successfully retrieved. Unsuccessful attempts were tallied separately; these included any attempt at pulling the flap out, or pushing the flap inward without successfully obtaining the food reward. The first attempt made by each monkey for each session was also recorded. Seventeen attempts were discarded from the push group, and 29 from the pull group due to the bias of the flap. In these cases the flap remained open and monkeys reached under the flap to gain access to the food. These were classified as opportunistic attempts, and only accounted for $2 \%$ of the data.

An independent observer, blind to the experimental design, coded five 5-minute sections of footage from both the push group and the pull group. As before, frequencies of both push and pull successes and attempts were tallied, resulting in a high correlation between observers (successful pulls: $r^{2}=0.98, \quad N=5, \quad p<0.001$; successful pushes: $\mathrm{r}^{2}=0.97, \mathrm{~N}=5, \mathrm{p}<0.001$; attempted pulls: $\mathrm{r}^{2}=0.94, \mathrm{~N}=5, \mathrm{p}<0.001$; attempted pushes: $\left.\mathrm{r}^{2}=0.92, \mathrm{~N}=5, \mathrm{p}<0.001\right)$. The difference between pushes and pulls was highly unambiguous, and tallying was therefore relatively straightforward.

\section{Results}

\section{Total Attempts}

The total number of attempts (both successful and unsuccessful manipulations of the apparatus) was analysed first. The analyses were divided into three main sections: sessions 1-8 (with the demonstration playing), follow-up session 9, and follow-up session 10 (both with no demonstration playing). As can be seen from Figure 2, not all individuals interacted with the apparatus during every session in sessions 1-8 (and in fact no individual interacted with the apparatus until session 2). However, by session 8, all four individuals from the smaller ("pull”) group had interacted with the apparatus, as had three individuals from the larger ("push") group. Each of these seven subjects also participated during sessions 9 and 10, with the exception of Scragg from the pull group who did not participate during session 9.

For each of these three sections (sessions 1-8, session 9 and session 10), discrimination ratios were calculated for each monkey, by taking the total number of correctly matched attempts (the type of manipulation observed) divided by the total 
number of attempts (Mottley \& Heyes, 2003). A summary of the results is provided in Table 1. In order to compare the proportions of the target actions in each group directly, further discrimination ratios were calculated measuring the proportion of pull responses made by each monkey by dividing the total number of pull attempts by the total number of attempts made. Due to the small and unequal number of subjects, Mann-Whitney U tests were used to analyse the data.

For sessions 1-8, the discrimination ratios of the proportion of pull responses made by each monkey were significantly higher in the pull group $\left(U_{4,3}=0, p<0.05\right.$, two-tailed). Thus the pull group pulled significantly more than those in the push group. Binomial tests were completed to look for differences between the number of pushes and pulls performed by each individual. All four subjects in the pull group pulled significantly more (Diamond, 94\% of her total attempts: Binomial test: $\mathrm{p}(132,8)<0.001$, two-tailed; Lottie, $98 \%$ of her total attempts: $\mathrm{p}(119,2<0.001$, twotailed; Beattie, $96 \%$ of her total attempts: $\mathrm{p}(26,1)<0.001$, two-tailed, and Scragg, $55 \%$ of her total attempts, $\mathrm{p}(279,232)<0.05$, two-tailed). All three subjects from the push group pushed significantly more (Kabul, $99.7 \%$ of his total attempts: $\mathrm{p}(383,1)<0.001$, two-tailed; Hope, $99 \%$ of his total attempts: $\mathrm{p}(79,1)<0.001$, two-tailed; Fergus, $100 \%$ of his total attempts: $\mathrm{p}(258,0)<0.001$, two-tailed $)$.

These same analyses were performed on sessions 9 and 10 separately. Only three of the four subjects from the pull group participated in session 9 (Scragg remained distant from the rest of the group throughout the session). All three subjects from the push group participated. As before, a significant difference was found between the proportion of pull responses between the groups (Mann Whitney $U_{3,3}=0$, $\mathrm{p}<0.05$, two-tailed). Binomial tests showed that all participating subjects from the pull group pulled significantly more than they pushed (Diamond, $100 \%$ of the total attempts: $p(7,0)=0.02$; Lottie, $99 \%$ of her total attempts: $p(116,1)<0.001$; Beattie, $98 \%$ of her total attempts: $p(83,2)<0.001)$. Two subjects from the push group pushed on $100 \%$ of their total attempts (Kabul: $p(71,0)<0.001$; Fergus: $p(129,0)<0.001)$. The remaining subject from the push group, Hope, pushed on $95 \%$ of his total attempts, $\mathrm{p}(19,1)<0.001$.

In Session 10 the pull group were still pulling significantly more than the push group (Mann-Whitney $\mathrm{U}_{4,3}=0, \mathrm{p}<0.05$, two-tailed). Three members of the pull group pulled significantly more (Diamond, $76 \%$ of her total attempts: $p(42,13)<0.001$; Lottie, $83 \%$ of her total attempts: $\mathrm{p}(120,25)<0.001$; Beattie, $95 \%$ of her total attempts: $\mathrm{p}(147,7)<0.001)$. Scragg actually pushed more often than she pulled, although this was not significant $(43 \%$ of her total attempts were pulls, $p(42,55)=0.223)$. All 3 members of the push group pushed on $100 \%$ of their total attempts (Kabul: $p(169,0)<0.001$; Hope: $p(50,0)<0.001$; Fergus: $p(88,0)<0.001)$. Figure 2 illustrates the difference between the two groups in the rate of push and pull responses across the sessions with the demonstration playing (Sessions 1-8), 24 hours post-demonstration (Session 9), and 5 days post-demonstration (Session 10). 


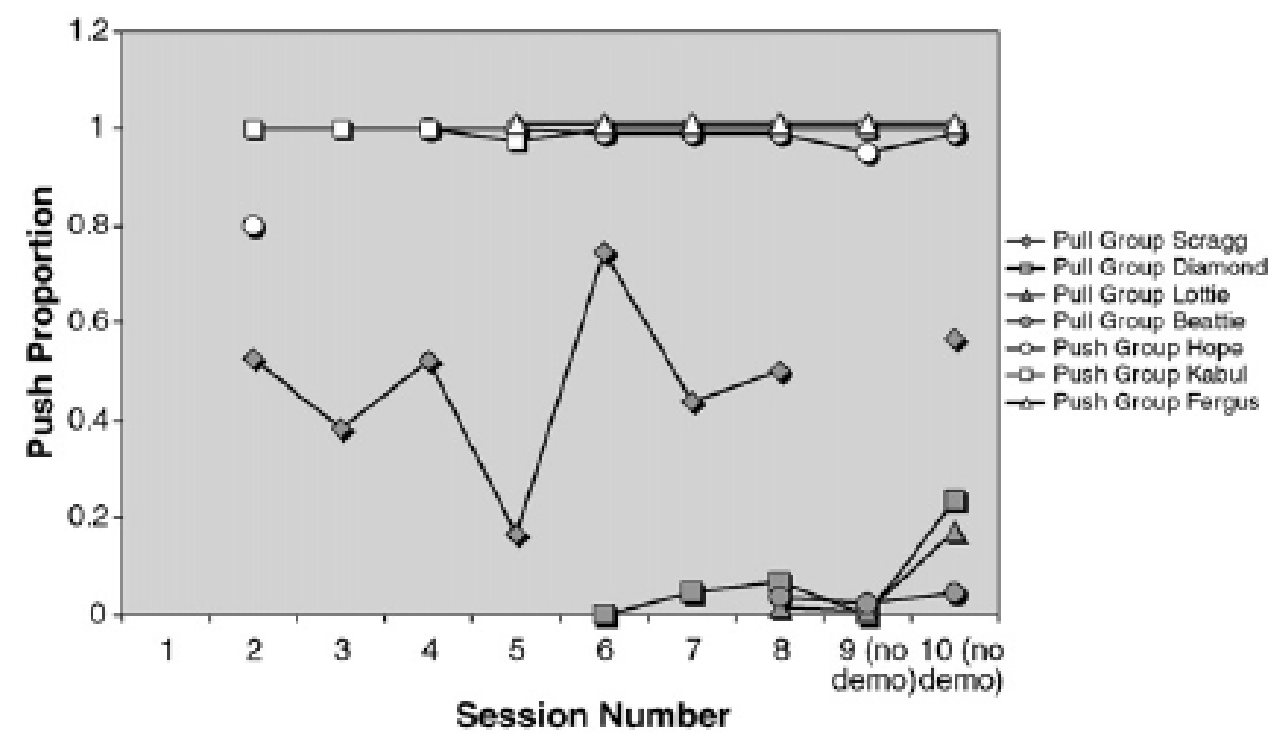

Fig. 2. Proportion of pushes to pulls for each subject, for all sessions. Demonstration clips were shown during sessions $1-8$. Session 9 was held $24 \mathrm{~h}$ after the demonstration footage had last been shown and session 10, 5 days later.

\section{Successful Attempts Only}

The same analyses were computed for successful attempts only (i.e. manipulations that resulted in the actor obtaining the food reward - see definition at the beginning of Results section). Thus the number of successful pull attempts was divided by the total number of successful attempts (pulls and pushes). Broadly speaking, this made little difference to the results, returning similar levels of significance for each test conducted (Mann-Whitney tests between groups and Binomial tests on individual data). The details of these extra analyses are therefore not reported here. The success rates of each subject, using both of the methods, are given in Table 2.

\section{First Responses}

As well as looking at the response totals, for each individual we also analysed their very first response in the first session towards the box. In all cases the very first response by each monkey corresponded to the observed response (Binomial test: $\mathrm{p}(7,0)<0.05$, two-tailed). 
Price, E. \& Caldwell, C. A. (2007). Artificially generated cultural variation between two groups of captive monkeys,

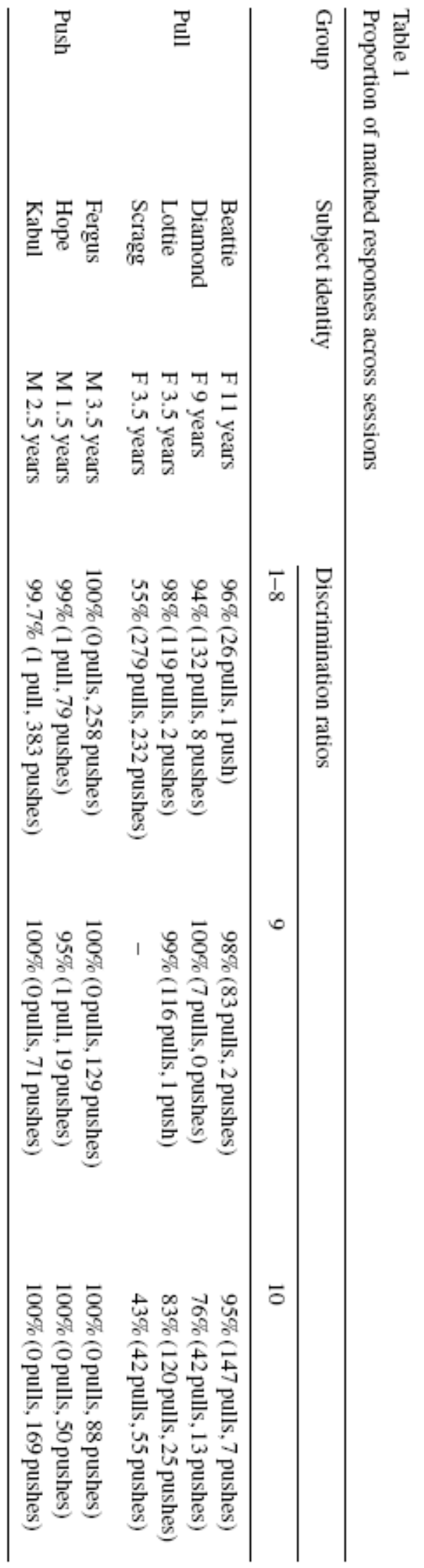




\section{Discussion}

As predicted, the group shown the pull demonstrations pulled significantly more than the group shown the push demonstrations. Six out of the seven subjects also consistently showed significantly higher proportions of matched to unmatched responses. The effect of the demonstration manipulation appeared to have a clear effect on the subjects' initial interactions with the apparatus, as all subjects matched the demonstrated behaviour on their very first attempt with the apparatus.

It should be noted that, since individuals could not be randomly assigned to conditions, the groups were unbalanced in terms of sex and age, which could have introduced a confound. Furthermore, it was necessary to test one group indoors and the other outdoors. However, it is unlikely that these differences between the groups would have caused the observed difference in action frequencies. Also, as it was not possible to separate the subjects during testing, it might be argued that differences between the groups were not due solely to the demonstration type, but also influenced by other group members. However, under this reasoning the subjects would still be displaying social learning. If one of the individuals learned through observation of the demonstration and then the behaviour subsequently spread throughout the group from that individual's actions, the results would be very intriguing indeed, as arguably two separate self-propagating behavioural traditions would have been created.

The effects of the demonstration persisted over time, even when the demonstration footage was no longer present. Six out of seven subjects still showed a significant tendency to match the demonstrated technique, even when tested 24 hours, and then five days, after the demonstration footage had been shown. The continued matching occurred despite all but two of the subjects at some point making use of the alternative method, and successfully obtaining the food reward when doing so (the exceptions were Fergus, who only ever pushed, and Hope, who made two unsuccessful pulls). These results therefore appear to be somewhat inconsistent with the expectations of Heyes (1993) and Galef (1995) regarding the effects of trial-anderror learning on socially learned behaviours.

It is of course entirely possible that, over an even longer period of time, our subjects would have eventually converged on the same solution (most likely the push technique). Although the effect that we found lasted over a longer period than has typically been tested in two-action studies, it would be extremely informative to run similar studies for even longer. Possibly over periods of months, or even years, group differences would extinguish. Longer term studies could investigate systematically whether groups showed trends towards converging on particular common solutions, or towards within-group conformity and between-group divergence. As mentioned in the introduction, Whiten et al. (2005) found that almost half of their chimpanzees behaved more like the group norm over time. The trends found in our study (see Figure 2) suggest that the colobus monkeys (at least those in the pull group) were gradually behaving less like the group norm, but the virtually ceiling level matching rates on most trials make such an observation somewhat difficult to interpret.

It is interesting to note that, of the few studies which have previously investigated the longevity of socially learned behaviours, initial contrasts in behaviour have generally been reported to extinguish fairly rapidly over trials, resulting in no difference between experimental groups. As noted in the introduction, Galef (1986) found that budgerigars matched their demonstrator for only two trials. There was no significant difference between the groups on the third, fourth or fifth trials. 
In Bugnyar and Huber's (1997) study of imitation in common marmosets, subjects were provided with a pendulum door that could be either pushed or pulled to gain access to a food reward. Observer monkeys watched a trained conspecific demonstrator pulling open the door, and although some observer monkeys showed a stronger tendency to use the demonstrated opening technique than control (nonobserver) monkeys, Bugnyar and Huber (1997) report that the initial preference of the observers disappeared over the course of five test trials, and all subjects converged on the simpler (push) alternative preferred by the non-observers.

Given our replication of the push/pull methodology, this latter study is particularly relevant. We might have expected the colobus monkeys in our study to converge on the push technique as Bugnyar and Huber's (1997) marmosets did. For our subjects, the pull technique required a bimanual manipulation of the apparatus, with the monkeys pulling the flap with one hand, whilst reaching in for the food reward with the other. Pushing, by contrast, was achieved with a single hand. It seems then that pullers may have been disadvantaged by their technique, yet three of the four monkeys in the pull group continued to pull significantly more than push through all of the sessions. Even Scragg, who used both techniques roughly equally (and therefore had ample opportunity to learn about the relative effectiveness of the two techniques) continued to make use of the pull technique on the final test session. As Table 2 shows, however, the apparent relative effort of the pull technique compared with the push alternative did not appear to translate into a clear discrepancy in the likelihood of each method resulting in a food reward.

These results can perhaps be compared to some of the variation in tool-use behaviours in natural primate populations. Out of several well studied populations of chimpanzees, clear differences have been found in the techniques used for ant-dipping (Whiten et al., 1999; Yamakoshi \& Myowa-Yamakoshi, 2004). The "pull-through" technique used in Gombe National Park, Tanzania is demonstrably more efficient that the "direct-mouthing" technique used by populations of monkeys in Taï, Côte d'Ivoire (e.g. see experiment conducted by Humle \& Matsuzawa, 2002, who used a human subject in order to directly compare the techniques). In the pull-through method, a long wand is used, and the ants are swept from the tool with the other hand. Despite its greater efficiency, this method is not used by the Taï group in preference of a slower, one-handed, method of sweeping the wand directly with the lips.

In terms of determining whether one of our two methods in this study was truly easier and/or more efficient, it would have been extremely useful to have had access to a third group of monkeys, which could have been exposed to the apparatus without any form of demonstration. This would have allowed us to determine whether our two experimental groups both showed a social learning effect, deviating away from the baseline group, or if the demonstration functioned only to produce a deviation from the baseline in one of the groups (i.e. by either inducing a very high level of conformity in the push group, or inducing otherwise unlikely pulling behaviour in the pull group). Unfortunately this was not possible for us as we only had access to two social groups of colobus monkeys. Nonetheless the clear contrast between the two groups' behaviour shows that there was certainly an effect of demonstration on the subjects. Furthermore, this social learning effect evidently had the power to create contrasting behavioural traditions in our two groups.

It may be the case that testing for social learning in groups of primates, rather than isolated individuals, in fact increases the time taken for behavioural variants to extinguish. The actions of group members may function as a repeat demonstration, influencing individuals to persist with a particular behaviour, counteracting the effects 
of their own experience through trial-and-error. However this is currently only a speculative suggestion based on the fact that ours and Whiten et al.'s (2005) studies were carried out in social groups. In order to test this, one would have to compare conditions in which subjects were tested either individually or in a social group, in order to find out in which of these conditions the socially learned variants corrupted more rapidly.

Regarding the social learning mechanism (or mechanisms) underlying the effects that we found, it is possible that imitation could have been involved. The discrimination ratios calculated for each subject are extremely high, in some cases demonstrating a virtually perfect copying rate. To our knowledge these represent some of the highest ratios seen thus far in the literature, and are certainly intriguing given the lack of knowledge regarding the cognitive abilities of the species in question. The two-action task was developed to rule out certain types of social learning, therefore it is relatively safe to say that the cause of the differences between groups is not due to local or stimulus enhancement, as both groups were equally drawn to the same part of the apparatus.

Members of the two groups also tended to adopt techniques closely matched to those they had observed. The push group pushed with one hand through the flap to remove the food, as seen in their videoclip. Also, the pull group consistently used the same pulling method as that shown on their tape, i.e. they pulled up on the corner of the flap, and then reached in with the other hand to retrieve the food. This was despite the fact that other effective alternatives were available, such as pulling from the screw handles which had been intentionally placed on the apparatus for that purpose (see method section). In the study carried out by Custance et al. (1999) with capuchin monkeys, they suggested that the idiosynchratic methods chosen by the subjects were suggestive of object-movement re-enactment (a form of emulation), rather than imitation. However, no such effect was found in the current study as monkeys in from the same demonstration groups were highly consistent in their preferred methods. Nonetheless, it remains a possibility that the results could be attributable to objectmovement re-enactment (or indeed response facilitation, e.g. Byrne \& Russon, 2001), rather than true imitation.

\section{Acknowledgments}

We are grateful for the cooperation of staff at Paignton Zoo, particularly Don, Lisa, and Julian. We also thank Kate Horton for coding data, and for help with transportation during data collection.

\section{References}

Bugnyar, T. and Huber, L., 1997. Push or pull: an experimental study on imitation in marmosets. Anim. Behav., 54: 817--831.

Byrne, R.W. and Russon, A.E., 2001. Learning by imitation: a hierarchical approach. Behav. Brain Sci., 21, 667--721.

Custance, D.M., Whiten, A. and Fredman, T., 1999, Social learning of artificial fruit processing in enculturated capuchin monkeys. J. Comp. Psychol., 113: 13--23.

Cook, M. and Mineka, S., 1989, Observation conditioning of fear to fear-relevant versus fear-irrelevant stimuli in rhesus monkeys. J. Abnorm. Psychol., 98: 448-459

Coussi-Korbel, S. and Fragaszy, D. M., 1995, On the relation between social dynamics and social learning. Anim. Behav., 50: 1441--1453. 
Davies, A.G. and Oates, J.F., 1994, Colobine Monkeys: Their Ecology, Behaviour and Evolution. Cambridge University Press, Cambridge.

Dawson, B.V. and Foss, B.M., 1965, Observational learning in budgerigars. Anim. Behav., 13: 470--474.

D’eath, R.B., 1998, Can video images imitate real stimuli in animal behaviour experiments? Biol. Rev., 73: 267--292.

Galef, B.G. Jr., 1995, Why behavior patterns that animals learn socially are locally adaptive. Anim. Behav., 49: 1325--1334.

Galef, B.G. Jr., 2003, "Traditional" foraging behaviours of brown and black rats (Rattus norvegicus and Rattus rattus). In: D.M. Fragaszy and S. Perry (Editors), The Biology of Traditions: Models and Evidence, Cambridge University Press, Cambridge, pp. 159--186.

Galef, B.G. Jr., Manzig, L.A. and Field, R.M., 1986, Imitation learning in budgerigars: Dawson and Foss 1965 revisited. Behav. Process., 13: 191--202.

Heyes, C.M., 1993, Imitation, culture and cognition. Anim. Behav., 46: 999--1010.

Hulme, T. and Matsuzawa, T., 2002, Ant-dipping among the chimpanzees of Bossou, Guinea, and some comparisons with other sites. Am. J. Primatol., 58: 133--148.

Ikebuchi, M. \& Okanoya, K. (1999). Male zebra finches and Bengalese finches emit directed songs to the video images of conspecific females projected onto a TFT display. Zool. Sci., 16: 63--70.

Laland, K.N. and Hoppitt, W., 2003, Do animals have culture? Evol. Anthropol., 12: 150--159.

Mottley, K. and Heyes, C., 2003, Budgerigars (Melopsittacus undulatus) copy virtual demonstrators in a two-action test. J. Comp. Psychol., 117: 363--370.

Panger, M.A., Perry, S., Rose, L., Gros-Louis, J., Vogel, E., MacKinnon, K.C. and Baker, M., 2002, Cross-site differences in foraging behaviour of white-faced capuchins (Cebus capucinus). Am. J. Phys. Anthropol., 119: 52--66.

Perry, S., Baker, M., Fedigan, L., Gros-Louis, J., Jack, K., MacKinnon, K.C., Manson, J.H., Panger, M., Pyle, K. and Rose, L., 2003, Social conventions in wild white-faced capuchin monkeys: evidence for traditions in a Neotropical primate. Curr. Anthropol., 44: 241--268.

Van Schaik, C.P., Ancrenaz, M., Borgen, G., Galdikas, B., Knott, C.D., Singleton, I., Suzuki, A., Utami, S.S. and Merrill, M., 2003, Orangutan cultures and the evolution of material culture. Science, 299: 102--105.

Voelkl, B. and Huber, L., 2000, True imitation in marmosets. Anim. Behav., 60: 195-202.

Walker, P., Murray, P., 1975, An assessment of masticatory efficiency in a series of anthropoid primates with special reference to the Colobinae and Ceropithecinae. In: R.H. Tuttle (Editor) Primate Functional Morphology and Evolution, Mouton, The Hague, pp. 151--194.

Whiten, A., Custance, D.M., Gomez, J.C., Texidor, P. and Bard, K.A., 1996, Imitative learning of artificial fruit processing in children (Homo sapiens) and chimpanzees (Pan troglodytes). J. Comp. Psychol., 110: 3--14.

Whiten, A., Goodall, J., Mcgrew, W.C., Nishida, T., Reynolds, V., Sugiyama, Y., Tutin, C.E.G., Wrangham, R.W. and Boesch, C., 1999, Cultures in chimpanzees. Nature, 399: 682--685.

Whiten, A., Goodall, J., Mcgrew, W.C., Nishida, T., Reynolds, V., Sugiyama, Y., Tutin, C.E.G., Wrangham, R.W. and Boesch, C., 2001, Charting cultural variation in chimpanzees. Behaviour, 138: 1481--1516. 
Price, E. \& Caldwell, C. A. (2007). Artificially generated cultural variation between two groups of captive monkeys, Colobus guereza kikuyuensis. Behavioural Processes, 74, 13-20.

Whiten, A., Horner, V. and de Waal, F.B.M., 2005, Conformity to cultural norms of tool use in chimpanzees. Nature, 437: 737--740.

Yamakoshi, G., Myowa-Yamakoshi, M., 2004, New observations of ant-dipping techniques in wild chimpanzaees at Bossou, Guinea. Primates, 45: 25--32. 